\title{
Failure Probability of Ceramic Coil Springs
}

\author{
S. Nohut, G. A. Schneider \\ Hamburg University of Technology, Institute of Advanced Ceramics, Denickestr. 15, D-21073 Hamburg, \\ Germany \\ Corresponding author : : Gerold A. Schneider \\ Tel : : $+49-(0) 40428783137$ \\ Fax : : +49-(0)40 428782647 \\ E-mail : g.schneider@tu-harburg.de
}

\begin{abstract}
Ceramic springs are commercially available and a detailed reliability analysis of these components would be useful for their introduction in new applications. In this paper an analytical and a numerical analyses of the failure probability for coil springs under compression is presented. Based on analytically derived relationships and numerically calculated results, fitting functions for volume and surface flaws will be introduced which provide the prediction of the failure probability of ceramic coil springs with different spring- and material parameters. As an example, typical mechanical properties for $\mathrm{Si}_{3} \mathrm{~N}_{4}$ are chosen. It is shown that surface flaws control the strength of the investigated springs.
\end{abstract}

Keywords: B. Failure analysis, C. Fracture, C. Strength, C. Mechanical Properties, Ceramic springs

\section{Introduction}

Springs are fundamental mechanical components which form the basis of many mechanical systems. Unlike other components, they undergo significant deformation when loaded and their compliance enables them to store readily recoverable mechanical energy. Thus springs must possess high strength against the applied force. For that reason, high strength materials such as high carbon steels have been and are still major materials for springs.

However, in recent years the increase of efficiency in many mechanical systems has become more important and this has introduced a new demand for the conservation of more energy in springs. In addition there is need of springs in different working conditions for example at high temperature, high wear rate or high corrosion rate. One way to satisfy these demands is the introduction of new materials for springs. Ceramics have this potential with excellent resistivity to heat, low density, high strength at high 
temperatures and better corrosion and wear resistances compared to other materials and therefore there is a growing interest in the fabrication of ceramic springs.

The loss coefficient is an important dimensionless material parameter in cyclic loading and plays an important role in the material selection for springs ${ }^{1}$. It is the fraction of mechanical energy loss in a stressstrain cycle. If a material is linear loaded elastically to a stress $\sigma$ with corresponding strain $\varepsilon$, it stores elastic strain energy per unit volume, $u=1 / 2 E \varepsilon^{2}$, where $E$ is the Young's Modulus. If the material is unloaded (after loading), it dissipates energy $\Delta u$. The loss coefficient $\eta$ per radian can be defined as $\eta=\frac{\Delta u}{2 \pi u}$

A high loss coefficient is desirable for damping vibrations while a low loss coefficient transmits energy more efficiently. On the other hand, since the minimum energy loss is desired, the material which is used for springs should have a low loss coefficient. A material property chart of the loss coefficient $\eta$ at $30{ }^{\circ} \mathrm{C}$ plotted against the Young's modulus $E$ is supplied for almost all kinds of material by Ashby ${ }^{2}$. Elastomers have the highest loss coefficient $(\eta=1)$ and advanced ceramics have the lowest with a four-order-ofmagnitude reduction compared to elastomers $\left(\eta=10^{-4}\right)$. High-carbon steels have just slightly higher loss coefficients than the ceramics.

As stated before, one of the new demands placed on springs arises from the working conditions where the springs are used. At this point, the materials nowadays used for springs have some limitations with respect to temperature, corrosion and wear. With ceramics such as $\mathrm{Al}_{2} \mathrm{O}_{3}, \mathrm{Si}_{3} \mathrm{~N}_{4}$ or $\mathrm{SiC}$, it is possible to work at high temperature up to approximately $1000^{\circ} \mathrm{C}$ which is not possible with most other materials. Sato et al. ${ }^{3}$ made some experiments and broke $\mathrm{Si}_{3} \mathrm{~N}_{4}$ ceramic springs at different temperatures. They observed no decrease in fracture stress until $1000^{\circ} \mathrm{C}$ and reported that the mean fracture stress at $1200^{\circ} \mathrm{C}$ was only slightly less than the values obtained at lower temperatures. Furthermore, Rubesa \& Danzer ${ }^{4}$ mentioned the importance of ejection velocity of springs in the technical applications and reported advanced ceramics as interesting alternative materials to high carbon steels for the spring production according to the analytically calculated ejection velocity.

There are few references on ceramic springs in the literature. Helical ceramic springs produced from sintered vitreous-bonded alumina were reported by Chironis ${ }^{5}$. Hamilton et al. $^{6}$ investigated the mechanical properties of helical ceramic springs manufactured from $\mathrm{MgO}$ partially stabilized zirconia. They measured the spring deflection versus applied load at room temperature and also at high temperatures and observed that the material successfully obeys Hooke's law also at elevated temperatures. 
Wright et al. ${ }^{7}$ investigated the effect on the surface quality of a polymer-ceramic suspension used for the fabrication $\mathrm{Al}_{2} \mathrm{O}_{3}$ springs and windings by extrusion. The strength of $\mathrm{Si}_{3} \mathrm{~N}_{4}$ coil springs at high temperatures up to $1000{ }^{\circ} \mathrm{C}$ and the influence of size on the strength of the springs by derivation of an equation to calculate the effective volumes of coil springs were examined by Sato et al. ${ }^{3}$ It was reported that the mean strength of coil springs decreases with increasing effective volume which can be applied in design calculation.

Ceramic springs are mostly manufactured by extrusion of a polymer-ceramic suspension ${ }^{7,8}$. The main steps of this method are preparation of the suspension, extrusion, debinding and finally sintering. The composition of the suspension, the working temperatures and the way of removal of diluents are important parameters in order to avoid surface defects and to obtain a valuable surface quality. Furthermore, there is also a study in the literature where the springs were produced by machining the zirconia tubes ${ }^{6}$.

Despite the advantageous properties of ceramics, there are not too many examples of ceramic springs in the industrial applications. Firstly, manufacturing of springs from ceramics is more expensive compared to other materials. Secondly and the major reason is the brittle failure of ceramics under tension. Since even a very small defect can cause the failure of the component and there is a scatter of strength values, ceramics are less reliable in construction from the point of view of strength compared with metals. However, we are not aware of any study in the literature which gives hints to the reliability of ceramic springs. Nevertheless we think that there is a need for such a reliability analysis to provide useful information to the user about the failure probabilities of springs with different spring- and material parameters under different loading conditions.

The main aim of this paper is an analytical and a numerical failure probability analyses of ceramic coil springs under compression. The mechanical properties of $\mathrm{Si}_{3} \mathrm{~N}_{4}$ ceramics will be used as an example. Firstly, the coil springs will be shortly introduced. It will be explained how to get series of springs which meet the user specifications with a wide range of values for spring constant $k$, maximum displacement $\delta_{\max }$ or maximum allowable force $F_{\max }$. Afterwards, the scaling of failure probability with spring- and material parameters will be established analytically by solving the Weibull distribution function with the theoretical stress distribution in the spring. Thereafter, a numerical analysis will be performed with the finite element software ABAQUS and the post processor $\mathrm{STAU}^{9}$ which takes into account the effect of boundary conditions, contact stresses and multiaxial loading. Finally fitting functions for volume flaws and surface flaws will be introduced which corrects the analytical results based on the numerical solutions. 
This makes it possible to predict the failure probability of ceramic springs with different spring geometry and material parameters.

\section{Coil springs}

There are several types of springs used for different applications. In this paper, the reliability of ceramic coil springs under compression load is studied since ceramics are much stronger and more reliable under compression than under tension. Their turns are not touching in the unloaded position and they need no attachment points. If these springs are not compressed beyond their elastic limit, they obey Hooke's law, which states that the force $F$ by which the spring is compressed is linearly proportional to the distance $x$ from its equilibrium length:

where $k$ is the spring constant of the spring.

$$
F=-k x
$$

There are four types of commonly used springs, namely plain, plain-ground, squared and squared-ground springs. They differ from each other by their ends ${ }^{1}$. Here the investigated spring type is a plain spring which is shown in Fig. 1. This type of spring is used because it is the easiest to be treated for the analytical failure probability calculation.

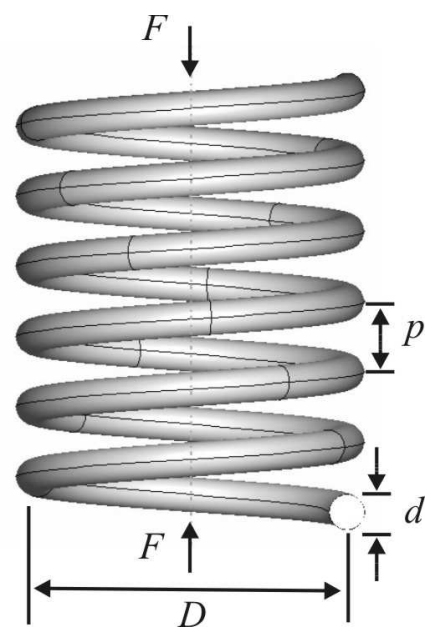

Fig. 1. Schematic representation of plain type coil spring with used spring parameters

The spring parameters shown in Fig. 1 are

$d \quad:$ wire diameter $[\mathrm{mm}]$

$D \quad$ : spring diameter $[\mathrm{mm}]$

$p \quad$ : pitch, which is the distance between the center of two coils [mm]

F $\quad$ : applied force $[\mathrm{N}]$ 
The spring index $C$ gives the ratio of the spring diameter to wire diameter and takes typically values between 3 and 12 .

$$
C=\frac{D}{d}, \quad 3 \leq C \leq 12
$$

$N_{a}$ gives the number of active coils which is equal to the total number of coils for plain type of coil springs. In this study, each spring will be specified by using the parameters $d, C$ and $N_{a}$. For example, $d 1 C 6 N_{a} 5$ means a spring with wire diameter of $d=1 \mathrm{~mm}$, spring diameter of $D=C \cdot d=6 \mathrm{~mm}$ and $N_{a}=$ 5 active coils.

In order to get a series of springs which supply the user a selection of different sizes and parameters, some initial spring parameters should be identified. First of all, initial values are given to $d, C$ and $N_{a}$. Then it is possible to calculate the spring constant $k$, and the solid length $l_{s}$ which is defined as the length of the spring when under sufficient load to bring all coils into contact with adjacent coils and additional load causes not further deflection, as reported by Hamrock et al. ${ }^{1}$

$$
\begin{gathered}
k=\frac{G d}{8 C^{3} N_{a}\left(1+\frac{0.5}{C^{2}}\right)} \approx \frac{G d}{8 C^{3} N_{a}} \\
l_{s}=d\left(N_{a}+1\right)
\end{gathered}
$$

where $G$ is the shear modulus of elasticity.

The next step is the assignation of pitch value, $p$ which is defined according to the desired displacement of the spring. After the pitch is assigned, it is possible to calculate the free length $l_{f}$ which is defined as the overall length of a spring in the unloaded position, maximum displacement of the spring, $\delta_{\max }$ and the maximum applied force $F_{\max }$ as given in Eq. (5), (6), and (7).

$$
\begin{aligned}
& l_{f}=p N_{a}+d \\
& \delta_{\text {max }}=l_{f}-l_{s} \\
& F_{\max }=\delta_{\max } k
\end{aligned}
$$

\section{Analytical scaling of failure probability}

Failure of ceramic materials is caused by unstable propagation of natural flaws existing in the material. Since there is a scatter in location, size and orientation of these flaws, the strength of ceramic components also scatters. In order to use ceramics as engineering materials, the strength has to be characterized. The 
most widely used expression for characterization is the cumulative distribution function proposed by Weibull ${ }^{10}$ in 1939. Since that time, this theory has become the one most widely used for application to the fracture of ceramic materials. The function depends on the "weakest-link-hypothesis" where the most dangerous flaw controls the strength. The most dangerous flaw is the flaw for which the most unfavorable combination of size, location and orientation in the stress field is obtained ${ }^{11}$. As a detailed derivation of the failure probability using Weibull statistics with multiaxial failure criteria is not the focus of this article, the reader is referred to the literature ${ }^{12-14}$.

In order to calculate the failure probability analytically, the theoretical stress distribution occurring in the spring is to be used. Here the helical spring form can be assumed as a straight wire which has the same stress distribution on cross-section and the same volume as the spring. When the spring is compressed with a force $F$ (see Fig. 1), torsional shear stress is produced due to torque on the cross-section (Fig. 2a). Additionally, a transverse ${ }^{1}$ (direct) shear stress appears (Fig. 2b) due to the bending of the wire because the spring is not a straight wire but is coiled with a pitch.

(a)

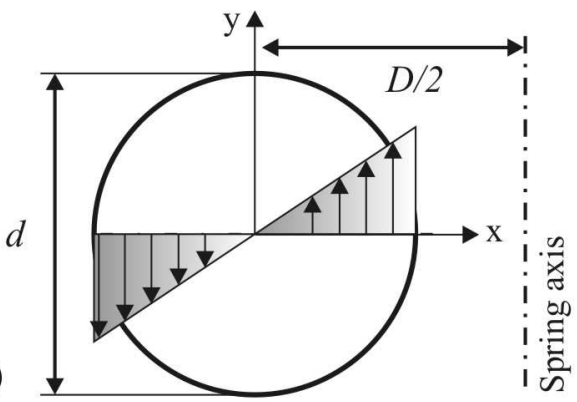

(b)

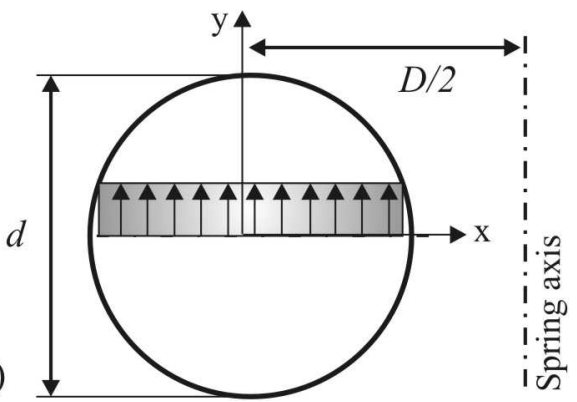

Fig. 2. Shear stresses acting on the cross-section of wire (a) torsional shear stress; (b) transverse (direct) shear stress. The z-direction is defined by the clockwise turning of $x-y$ system

The maximum torsional shear stress on the cross-section of the wire illustrated in Fig. 2a can be calculated by Eq. (8),

$$
\tau_{t, \max }=\frac{8 F D}{\pi d^{3}}
$$

The transverse shear stress shown in Fig. $2 b$ is constant over the cross-section of the wire and can be expressed for a circular cross section

$$
\tau_{d}=\frac{4 F}{\pi d^{2}}=\frac{1}{2 C} \tau_{t, \max }
$$

The change in the torsional shear stress can be described as follows using the cylindrical coordinate system given in Fig. 3a, 


$$
\tau_{t}(\mathrm{r})=\tau_{t, \max }\left(\frac{r}{d / 2}\right)
$$

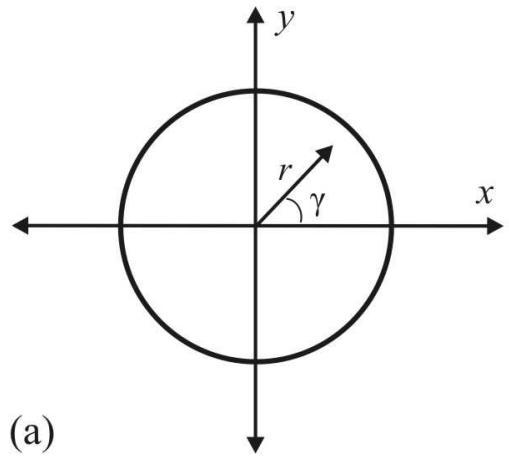

(b)

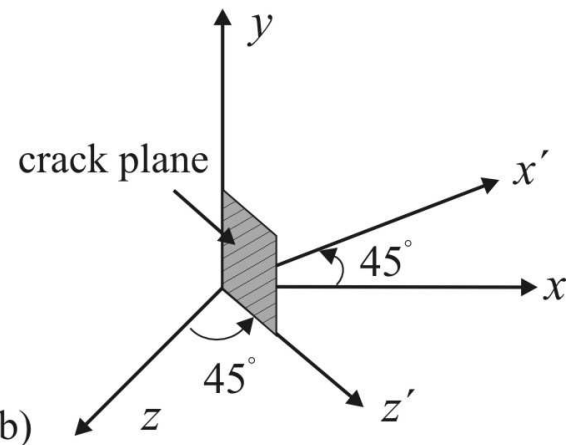

Fig. 3. (a) Cylindrical coordinate system used for the introduction of the stress tensor on the cross-section of the wire (b) Rotation of the coordinate system by $45^{\circ}$ around $y$-axis

The stress tensor in the volume of the springs in the $\mathrm{x}, \mathrm{y}, \mathrm{z}$ - coordinate system (see Fig.2) can be written as follows;

$$
\sigma(r, \gamma)=\left[\begin{array}{ccc}
0 & 0 & -\tau_{t}(r) \sin (\gamma) \\
0 & 0 & \tau_{t}(r) \cos (\gamma)+\tau_{d} \\
-\tau_{t}(r) \sin (\gamma) & \tau_{t}(r) \cos (\gamma)+\tau_{d} & 0
\end{array}\right]
$$

Here the normal stresses in the z-direction are assumed to be zero (plane stress approximation) since the appeared longitudinal stresses due to the pure torsion are very small for materials like steels and ceramics which have high shear moduli compared to rubber-like materials and hence can be neglected ${ }^{15,16}$. In this part for the sake of simplicity, only the cracks which are lying in one plane will be investigated. Lets us assume a crack lying on the $z^{\prime}-y$ plane whose normal is in the direction of $x^{\prime}$ (see Fig. 3b). The normal stress acting on the crack which is found by rotating the stress tensor given in Eq. (11) by $45^{\circ}$ around the $y$-axis is equal to

$$
\sigma_{n}(r, \gamma)=\tau_{t}(r) \sin (\gamma)=\left\{\begin{array}{l}
\tau_{t, \max }\left(\frac{r}{d / 2}\right) \sin (\gamma) \text { for } 0 \leq \gamma \leq \pi \\
-\tau_{t, \max }\left(\frac{r}{d / 2}\right) \sin (\gamma) \text { for } \pi \leq \gamma \leq 2 \pi
\end{array}\right.
$$

The failure probability $P_{F, V}$ of a ceramic component containing volume flaws can be calculated according to the normal stress criterion as follows

$$
P_{F, V}\left(\sigma_{n}\right)=1-\exp \left[-\frac{1}{V_{0}} \int_{V}\left(\frac{\sigma_{n}(r, \gamma)}{\sigma_{0}}\right)^{m} d V\right]
$$


where $m$ is the Weibull modulus, $\sigma_{0}$ is the cumulative mean stress and $V_{0}$ is the unit volume containing an average number of flaws. The normal stress $\sigma_{n}(r, \gamma)$ can be written as

$$
\sigma_{n}(r, \gamma)=\sigma^{*} g(r, \gamma)
$$

where $\sigma^{*}$ is the reference stress (e.g. maximum principal stress in the component), $g(r, \gamma)$ is the geometry function. It is practical to introduce an effective volume $V_{\text {eff }}$ which can be described as the volume of tensile-test rods which show the same distribution of strength as the original component and is dependent on the size of component, its stress distribution and the properties of the assumed crack population.

$$
V_{\text {eff }}=\int_{V} g^{m} d V
$$

When we put Eq. (14) and (15) into Eq. (13) we get:

$$
P_{F, V}\left(\sigma^{*}\right)=1-\exp \left[-\frac{V_{\text {eff }}}{V_{0}}\left(\frac{\sigma^{*}}{\sigma_{0}}\right)^{m}\right]
$$

Because only very small failure probabilities $\left(P_{F, V}<<1\right)$ are of practical significance, $P_{F, V}$ can be approximated using a Taylor expansion

$$
P_{F, V}\left(\sigma^{*}\right)=1-\exp \left[-\frac{V_{\text {eff }}}{V_{0}}\left(\frac{\sigma^{*}}{\sigma_{0}}\right)^{m}\right] \approx\left[\frac{V_{\text {eff }}}{V_{0}}\left(\frac{\sigma^{*}}{\sigma_{0}}\right)^{m}\right] \text { for } P_{F, V}<1
$$

When we decompose the stress field given in Eq. (12) as given in Eq. (14), we get

$$
\begin{gathered}
\sigma^{*}=\tau_{t, \max } \\
g(r, \gamma)=\left\{\begin{array}{l}
\left(\frac{r}{d / 2}\right) \sin (\gamma) \text { for } 0 \leq \gamma \leq \pi \\
-\left(\frac{r}{d / 2}\right) \sin (\gamma) \text { for } \pi \leq \gamma \leq 2 \pi
\end{array}\right.
\end{gathered}
$$

The determination of the effective volume $V_{\text {eff }}$ by Eq. (15) leads

$$
\begin{aligned}
V_{\text {eff }} & =\int_{V}\left[\left(\frac{r}{d / 2}\right) \sin (\gamma)\right]^{m} d V=2 \int_{L=0}^{\pi D N_{a}} \int_{\gamma=0}^{\pi} \int_{r=0}^{d / 2} r^{m}\left(\frac{2}{d}\right)^{m}(\sin \gamma)^{m} r d r d \gamma d L \\
& =\frac{\pi}{2(m+2)} d^{3} C N_{a} \int_{\gamma=0}^{\pi}(\sin \gamma)^{m} d \gamma=\frac{2 V_{\text {spring }}}{\pi(m+2)} \int_{\gamma=0}^{\pi}(\sin \gamma)^{m} d \gamma
\end{aligned}
$$

where we introduced the volume of the spring :

$$
V_{\text {spring }}=\frac{\pi^{2}}{4} d^{3} C N_{a}
$$

Inserting the derived $\sigma^{*}$ and $V_{\text {eff }}$ into Eq. (16) results in 


$$
P_{F, V}\left(\tau_{\mathrm{t}, \max }\right)=1-\exp \left(-\frac{4 S_{V}(m) V_{\text {spring }}}{\pi^{2} V_{0}}\left(\frac{\tau_{\mathrm{t}, \max }}{\sigma_{0}}\right)^{m}\right)
$$

where $S_{V}(m)$ is a factor for the volume flaws which is dependent on Weibull modulus $m$ and equal to

$$
S_{V}(m)=\frac{\pi}{2(m+2)} \int_{\gamma=0}^{\pi}(\sin \gamma)^{m} d \gamma
$$

The solution of the integral given in Eq. (22) involves the gamma function $(I)$ and is not given here explicitly. When the factor $S_{V}(m)$ is known, the failure probability of the ceramic springs due to volume flaws with different spring- and material parameters under desired loading can be calculated. In this part, for simplicity the cracks in one specific plane were investigated. Actually, in the material there are cracks oriented in all directions. Therefore, in the next part $S_{V}(m)$ will be calculated numerically by using the normal stress as failure criterion.

\section{Procedure of numerical failure probability calculation}

The calculation of failure probability of multiaxially loaded ceramic springs is done in two steps, stress analysis using the finite element software ABAQUS and statistical analysis using a finite element postprocessor STAU (STatistische AUswertung) which was developed by the Probabilistic Group at the IZSM at Karlsruhe University in cooperation with several partners ${ }^{9}$. Mechanical properties which are typical for $\mathrm{Si}_{3} \mathrm{~N}_{4}$ materials are chosen. During the stress analysis, $\mathrm{Si}_{3} \mathrm{~N}_{4}$ ceramic material is assumed to be isotropic elastic with Young's Modulus $E=300 \mathrm{GPa}$, and Poisson's ratio $v=0.25$. In the calculation of the failure probability, the normal stress criterion ${ }^{9}$ (Mode-I failure criterion) is applied.

\section{Results}

The failure probability of a spring due to volume flaws with given spring- and material parameters under desired loading can be calculated if $S_{V}(m)$ is known. In this part, the failure probability of springs will be computed with ABAQUS and STAU and from these results; the factor $S_{V}(m)$ will be obtained through Eq.

(21). The numerically calculated $S_{V}(m)$ values for different $m$ and $C$ values are given in Table 1.

\begin{tabular}{|c|c|c|c|c|c|}
\hline $\mathbf{C}$ & $\mathbf{8}$ & $\mathbf{1 0}$ & $\mathbf{1 2}$ & $\mathbf{1 4}$ & $\mathbf{1 6}$ \\
\hline $\mathbf{6}$ & $2.48 \times 10^{-3}$ & $1.36 \times 10^{-3}$ & $7.17 \times 10^{-4}$ & $4.54 \times 10^{-4}$ & $2.73 \times 10^{-4}$ \\
\hline $\mathbf{7}$ & $2.92 \times 10^{-3}$ & $1.61 \times 10^{-3}$ & $8.57 \times 10^{-4}$ & $5.39 \times 10^{-4}$ & $3.26 \times 10^{-4}$ \\
\hline $\mathbf{8}$ & $3.31 \times 10^{-3}$ & $1.85 \times 10^{-3}$ & $1.00 \times 10^{-3}$ & $6.24 \times 10^{-4}$ & $3.76 \times 10^{-4}$ \\
\hline $\mathbf{9}$ & $4.04 \times 10^{-3}$ & $2.33 \times 10^{-3}$ & $1.26 \times 10^{-3}$ & $8.76 \times 10^{-4}$ & $5.71 \times 10^{-4}$ \\
\hline $\mathbf{1 0}$ & $4.66 \times 10^{-3}$ & $2.76 \times 10^{-3}$ & $1.55 \times 10^{-3}$ & $1.08 \times 10^{-3}$ & $7.19 \times 10^{-4}$ \\
\hline
\end{tabular}


During the calculations, it was observed that the factor $S_{V}(m)$ also depends on the spring index $C$. This dependence arises from the effect of the transverse shear stress on the combined shear stress occurring on the cross-section of wire. Furthermore, the increase in $C$ increases the contact area between the coils in the FEM analysis. The analytical solution does not take into account these effects. By fitting the results given in Table $1, S_{V}(m, C)$ is obtained as a function of $m$ and $C$ as given below:

$$
S_{V}(m, C)=\exp (0.17036 C-0.27476 m-4.87303)
$$

The final form of the failure probability of the coil spring is

$$
P_{F, V}\left(\tau_{t, \max }\right)=1-\exp \left(-\frac{4 \exp (0.17036 C-0.27476 m-4.87303) V_{\text {spring }}}{\pi^{2} V_{0}}\left(\frac{\tau_{\mathrm{t}, \max }}{\sigma_{0}}\right)^{m}\right)
$$

for $6 \leq C \leq 10,8 \leq m \leq 16,1 \leq d \leq 4$ and $4 \leq N_{a} \leq 8$. Eq. (24) is valid for all materials. The Weibull parameters $m$ and $\sigma_{0}$ of the material can be directly put into the equation. The elastic properties $E$ and $v$ of the material affect $\sigma_{0}$ in a load-controlled scheme and $\tau_{t, \max }$ in a displacement-controlled scheme.

In order to get a feeling for the reliability of a typical coil spring, we compare the effective volumes of the bend bars and tensile specimens with that of the spring having the same volume. For a prescribed failure probability, the strengths of two components with effective volumes $V_{\text {eff, }, 1}$ and $V_{\text {eff,2 }}$ have a relationship as given below.

$$
\frac{\sigma_{1}}{\sigma_{2}}=\left(\frac{V_{\text {eff }, 2}}{V_{\text {eff }, 1}}\right)^{1 / m}
$$

Here a 4-point-bending test (4PB), 3-point-bending test (3PB), tensile test (TT) and a spring test (ST) are modeled. For the 4PB, 3PB and TT, according to the norm ${ }^{17}$, specimens with a length of $25 \mathrm{~mm}$, height of $1.5 \mathrm{~mm}$ and width of $2 \mathrm{~mm}$ are used, corresponding to a volume of $75 \mathrm{~mm}^{3}$. For the 4-point-bending test the support distance of loading member is $10 \mathrm{~mm}$ and of support member is $20 \mathrm{~mm}$. For the spring test, the spring $d 1 C 6 N_{a} 5$ with a volume of $74 \mathrm{~mm}^{3}$ is selected because it has approximately the same volume as the bend bar and tensile specimens. All four tests are modeled under loading conditions which give the prescribed failure probability taken here as $P_{F, V}=10^{-4}$ due to volume flaws with $\sigma_{0}=750 \mathrm{MPa}$ and $m=12$. The maximum principal stress occurring in all tests is recorded as the reference stress, $\sigma^{*}$. Afterwards, the effective volumes are calculated with STAU. In Fig. 4, the reference stresses versus effective volume values for all 4 tests are represented. 


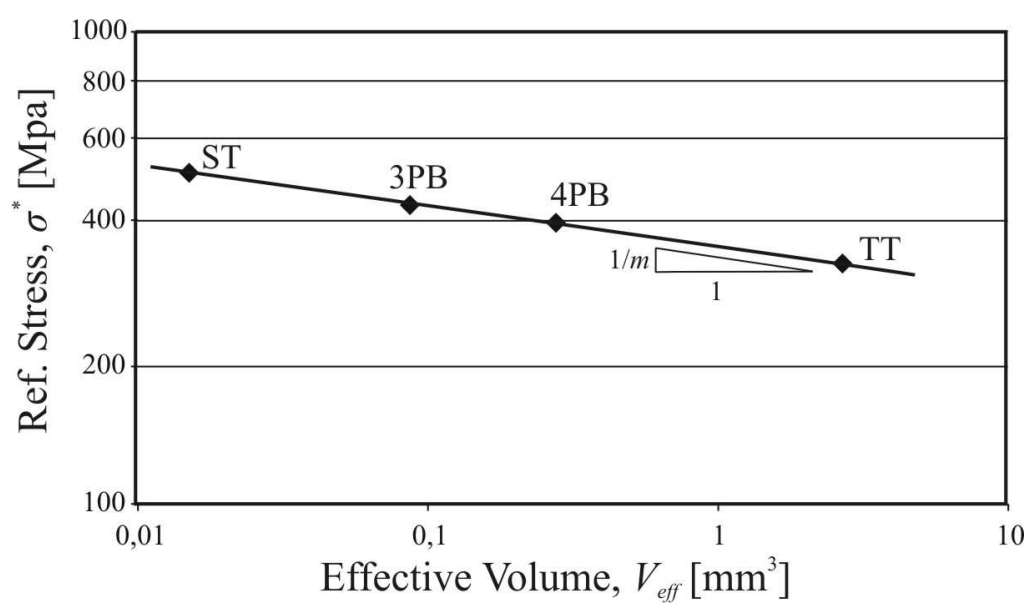

Fig. 4. Calculated reference stress, $\sigma^{*}$ versus effective volume, $V_{\text {eff }}$ values for 4-point-bending test, 3-point-bending test, tensile test and spring test for a prescribed failure probability of $P_{F, V}=10^{-4}$ due to volume flaws

The slope of the curve given in Fig. 4 is 0.0847 which is less than $2 \%$ close to the analytical value for $m=$ 12. The amazingly small effective volume for the tensile test specimen is due to the isotropic oriented crack planes.

The effective volume of the investigated coil spring is very small of the order of $10^{-2} \mathrm{~mm}^{3}$. Therefore, it is very probable that the failure of springs is due to the surface flaws. All calculations performed for the volume flaws will be now repeated for the surface flaws. In Table $2, S_{S}(m, C)$ values which are used in order to estimate the failure probability due to surface flaws are given for different $m$ and $C$ values.

\begin{tabular}{|c|c|c|c|c|c|}
\hline $\mathbf{C}$ & $\mathbf{8}$ & $\mathbf{1 0}$ & $\mathbf{1 2}$ & $\mathbf{1 4}$ & $\mathbf{1 6}$ \\
\hline $\mathbf{6}$ & $1.17 \times 10^{-1}$ & $9.31 \times 10^{-2}$ & $6.46 \times 10^{-2}$ & $5.23 \times 10^{-2}$ & $3.56 \times 10^{-2}$ \\
\hline $\mathbf{7}$ & $1.29 \times 10^{-1}$ & $1.06 \times 10^{-1}$ & $7.94 \times 10^{-2}$ & $6.23 \times 10^{-2}$ & $4.30 \times 10^{-2}$ \\
\hline $\mathbf{8}$ & $1.37 \times 10^{-1}$ & $1.14 \times 10^{-1}$ & $9.15 \times 10^{-2}$ & $7.12 \times 10^{-2}$ & $5.04 \times 10^{-2}$ \\
\hline $\mathbf{9}$ & $1.47 \times 10^{-1}$ & $1.29 \times 10^{-1}$ & $1.11 \times 10^{-1}$ & $9.29 \times 10^{-2}$ & $7.45 \times 10^{-2}$ \\
\hline $\mathbf{1 0}$ & $1.51 \times 10^{-1}$ & $1.41 \times 10^{-1}$ & $1.34 \times 10^{-1}$ & $1.13 \times 10^{-1}$ & $9.41 \times 10^{-2}$ \\
\hline
\end{tabular}

Table2: Numerical calculated $S_{S}(m, C)$ for different Weibull modulus $m$ and spring index $C$ values

After fitting the values in Table 2, the $S_{S}(m, C)$ function can be formulated as

$$
S_{S}(m, C)=\exp (0.31872 C-0.18989 m-2.35129)
$$

The failure probability of the ceramic coil springs due to the surface flaws can be written as

$$
P_{F, S}\left(\tau_{\mathrm{t}, \max }\right)=1-\exp \left(-\frac{S_{S}(m, C) S_{\text {spring }}}{\pi^{2} S_{0}}\left(\frac{\tau_{\mathrm{t}, \max }}{\sigma_{0}}\right)^{m}\right)
$$

where $S_{0}$ is the unit surface area and $S_{\text {spring }}$ is the total surface area of the spring and equal to

$$
S_{\text {spring }}=\pi^{2} d^{2} C N_{a}
$$

For the same specimens and same spring used before, the reference stress $\sigma^{*}$ and the effective surface $S_{\text {eff }}$ values for a prescribed failure probability of $P_{F, S}=10^{-4}$ due to surface defects are given in Fig. 5. When 
the surface flaws are taken into account, the effective surface of the ceramic coil spring is higher than the three-point bending specimen. Comparing Fig. 4 and Fig. 5, it can be concluded that the ceramic springs fail due to surface flaws.

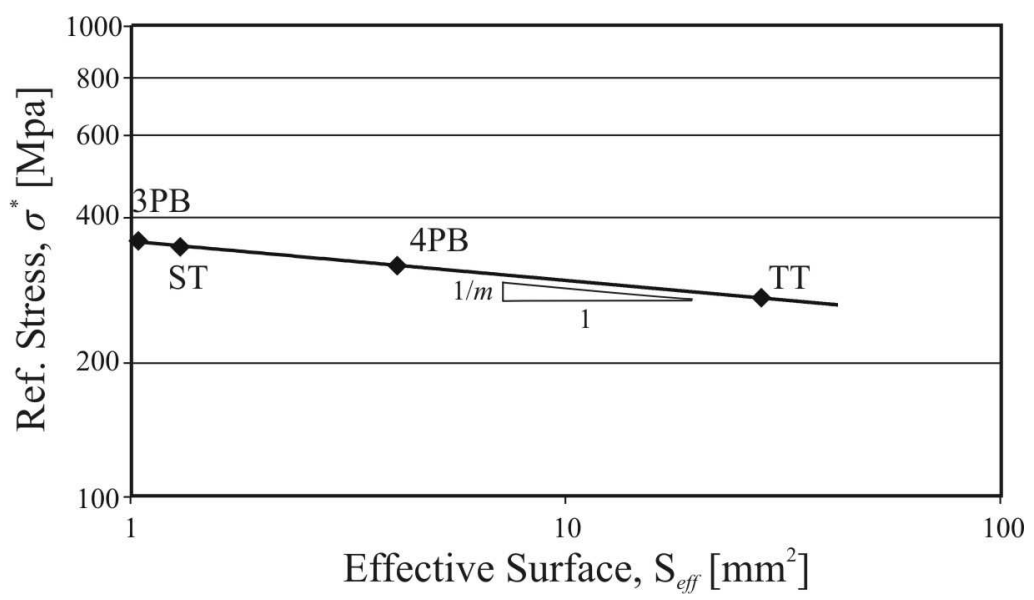

Fig. 5. Calculated reference stress, $\sigma^{*}$ versus effective surface, $S_{\text {eff }}$ values for 4-point-bending test, 3-point-bending test, tensile test and spring test for a prescribed failure probability of $P_{F, S}=10^{-4}$ due to surface flaws

Proof-Test

A proof test assesses whether a given ceramic component can survive under conditions similar to or worse than what would be expected in service. The proof test is applied before placing the part in service in order to improve reliability and increase the likelihood that it will survive over its intended service life. During the proof test with ceramic springs, the springs will be loaded with a stress which is larger than the desired stress in service and those springs with low strength and containing large defects can be eliminated. In addition, the proof test should be of short duration to avoid or reduce damage due to subcritical crack growth. Therefore, the loading- and unloading rate in proof test should be high.

Applying a proof test with a stress $\sigma_{P}^{*}$ which is larger than maximum stress $\sigma_{C}^{*}$ occurring in service, all the springs with strength $\sigma_{C}^{*}<\sigma_{P}^{*}$ will fail. The new failure probability $G\left(\sigma_{C}^{*}\right)$ of a spring which is loaded in the service with a stress higher than $\sigma_{P}^{*}$ will be calculated as ${ }^{18}$

$$
G\left(\sigma_{C}^{*}\right)=1-\exp \left[\frac{V_{\text {eff }}}{V_{0}}\left(-\left(\frac{\sigma_{C}^{*}}{\sigma_{0}}\right)^{m}+\left(\frac{\sigma_{P}^{*}}{\sigma_{0}}\right)^{m}\right)\right]
$$

Putting the Eq. (21) into Eq. (29) gives 
Journal of the European Ceramic Society 29[6] (2009) 1013-1019

doi:10.1016/j.jeurceramsoc.2008.08.012

$$
G\left(\sigma_{C}^{*}\right)=1-\exp \left(-\frac{4 S_{V}(m) V_{\text {spring }}}{\pi^{2} V_{0}}\left(-\left(\frac{\sigma_{C}^{*}}{\sigma_{0}}\right)^{m}+\left(\frac{\sigma_{P}^{*}}{\sigma_{0}}\right)^{m}\right)\right)
$$

Replacing the $\sigma_{C}^{*}$ and $\sigma_{P}^{*}$ by the corresponding forces $F_{C}$ and $F_{P}$ according to Eq. (8) and Eq. (18) gives:

$$
G\left(F_{C}\right)=1-\exp \left(-\frac{S_{V}(m) V_{\text {spring }} 8^{m} C^{m}}{\pi^{2+m} d^{2 m} V_{0} \sigma_{0}^{m}}\left(-\left(F_{C}\right)^{m}+\left(F_{P}\right)^{m}\right)\right)
$$

The same procedure can be applied for the surface flaws by using Eq. (27)

\section{Conclusion}

The principal scaling of the failure probability in regard to the volume of the spring and the reference stress was derived by calculating the effective volume analytically for one selected crack plane orientation. The generalization to arbitrarily oriented crack planes was performed numerically by using ABAQUS and STAU and is expressed by the introduction of fitting functions $S_{V}(m, C)$ and $S_{S}(m, C)$ for volume and surface flaws, respectively. Comparison of the failure probability of the spring with 4-point, 3-point and tensile test bars shows that the effective volume of spring is extremely small $\sim 10^{-2} \mathrm{~mm}^{3}$. The corresponding calculation for surface flaws clearly shows that surface flaws are strength controlling for ceramic coil springs.

\section{Acknowledgement}

The authors acknowledge support by DFG Graduiertenkollegs "Kunst und Technik". 
Journal of the European Ceramic Society 29[6] (2009) 1013-1019

doi:10.1016/j.jeurceramsoc.2008.08.012

\section{References}

1. Hamrock, J. B., Jacobson, B. O. \& Schmid, S. R., Fundamentals of machine elements, WCB/McGraw-Hill, Singapore, 1999.

2. Ashby, M. F., Materials selection in mechanical design, $3^{\text {rd }}$ edn. Elsevier, 2005.

3. Sato, A., Taguchi, R. K., Adachi, R. \& Nakatani, M. A., A study on strength characteristics of $\mathrm{Si}_{3} \mathrm{~N}_{4}$ coil springs. Fat. \& Frac. Eng. Mat. Struc., 1996, 19, 529-537.

4. Rubesa, D. \& Danzer, R., Die maximale Geschwindigkeit, auf die ein Körper durch eine Feder beschleunigt werden kann. Forschung im Ingenieurwesen - Engineering Research Bd. 61, 1995, 10 $249-254$

5. Chironis, N. P., Spring design and application. Mc-Graw-Hill, New York, 1961, pp.86

6. Hamilton, T., Gopal, M., Atchley, E. \& Smith, J. E., Experimental investigation on the mechanical performance of helical ceramic springs. J. Mat. Sci., 2003, 38, 3331-3335

7. Wright, J. K., Thomson, R. M. \& Evans, J. R. G., On the fabrication of ceramic windings. J. Mat. Sci., 1990, 25, 149-156

8. Coronel, J., Endkonturnahe Formgebung keramischer Bauteile über pulverbeladene Wachssuspension. Dissertation, Technische Universität Hamburg-Harburg, 2004,

9. Brückner-Foit, A., Heger, A., Heiermann, K., Hülsmeier, P., Mahler, A., Mann, A. \& Ziegler, C., STAU 5 - User's Manual - A post processor for a finite element program to calculate the failure probability under thermal schock loading. Institut für Materialforschung II, Karlsruhe, 2005

10. Weibull, W., A statistical theory of the strength of materials. Ingenioersvetenskaps-akademiens Handl. Stockholm, 1939, 151, 1-45

11. Bergman, B., On the estimation of the Weibull modulus. J. Mat. Sci., 1984, 3, 689-692

12. Batdorf, S. B., \& Crose, J. G., A statistical theory for the fracture of brittle structures subjected to nonuniform stress, J. Appl. Sci., 1974, 41, 459-461

13. Batdorf, S. B., \& Heinisch, H. L., Weakest link theory reformulated for arbitrary fracture criterion, $J$. Am. Ceram. Soc., 1978, 61, 355-358

14. Matsuo, Y., Kitakami, K. \& Kimura, S., A new theory of non-destructive inspection based on fracture mechanics and fracture statistics, in Fracture Mechanics of Ceramics, 10, R. C. Bradt et. al., Eds., Plenum Press, New York, 1992, pp. 317-327 
Journal of the European Ceramic Society 29[6] (2009) 1013-1019

doi:10.1016/j.jeurceramsoc.2008.08.012

15. Timoshenko, S., Strength of materials, Part II-Advanced theory and problems, Robert E. Krieger Publishing Company, Florida, 1983

16. Timoshenko, S., Strength of materials, Part I-Elementary theory and problems, Robert E. Krieger Publishing Company, Florida, 1976

17. ASTM C 1161 - 02c. Standard test method for flexural strength of advanced ceramics at ambient temperature.

18. Munz, D. and Fett T., Ceramics - Mechanical Properties, failure behaviour, materials selection. Springer-Verlag (Springer series in materials science; v.36), Berlin, 1999 